\title{
DESIGN AND CONSTRUCTION OF A DRONE WITH GPS TRACKING, COLLISION AVOIDANCE, SURVEILLANCE VIA AIRBORNE CAMERA AND OBJECT TRACKING
}

\author{
Zaharias Gerostathis \\ Hellenic Naval Academy \\ Piraeus, Athens, Greece
}

\author{
Georgios Thomas \\ Hellenic Naval Academy \\ Piraeus, Athens, Greece
}

\author{
Andreas Tsigopoulos \\ Hellenic Naval Academy \\ Piraeus, Athens, Greece
}

\author{
Kostas Harisis \\ Hellenic Naval Academy \\ Piraeus, Athens, Greece
}

\begin{abstract}
Unmanned Aerial Vehicles (UAVs) often called drones, are becoming increasingly important for military and security operations. This paper presents the analysis of the design and construction of a flying autonomous robotic vehicle or drone. The drone constructed is a quadcopter and in its final form will be able to take off, swing holding steady its position, move in space and avoid obstacles according to a predefined pattern as well as report its position in space via GPS tracking. The drone uses Arduino microcontroller as its flight controller that communicates directly with the drone's sensors and is responsible for controlling them. The drone also carries a camera that can be operated manually or automatically to monitor and lock onto a target, based on its color, or just survey the field of operation. The camera can also move independently of the drone based on the commands received to the ESP-32 microcontroller from the user's computer, where the video processing takes place.
\end{abstract}

Keywords- Quadcopter, Object Tracking, GPS Tracking, Arduino, Collision Avoidance

\section{INTRODUCTION}

The research and development of drones dates back to the beginning of the First World War. There was a continuous effort for development during the interwar period, the Second World War and afterwards, which has led to the creation of cruise missiles (Harpoon, Tomahawk), air targets for military training and finally to the family of Unmanned Air Vehicles (UAVs) (Jackson et al., 2008). Although today there is a wide variety of uses for the UAVs, perhaps the most significant is still, the one for the military (Guilmartin, 2019). In this paper, the complete analysis of the design and construction of a drone will be presented. The drone is a quadcopter and can move in space avoiding obstacles while reporting its position via GPS tracking and is able to lock on a target and follow it.

\author{
Argyris Stassinakis \\ National and Kapodistrian University of Athens \\ Athens, Greece
}

The rest of the paper is organized as follows. The designing and making of the drone frame as well as the camera mounting system is presented in section II. Section III refers to the electronic parts and the programming algorithms used. Experimental results will be discussed in section IV. Concluding remarks are given in section V.

\section{Design AND CONSTRUCTION}

\section{A. Drone Frame}

For the construction of the frame we used aluminium as the main material in order to achieve the desired durability and strength. The two axes where the motors are mounted $(50 \mathrm{~cm}$ long each), are made from aluminium channel section and they are pacted in an aluminium sheet $(200 \times 140 \times 1.5 \mathrm{~mm})$. The electronic parts are placed on an aluminium sheet (200x $140 \times 3 \mathrm{~mm})$ and the battery is situated in an aluminium made box underneath the whole construction. All the pieces are connected with screws so they can be easily dismantled.

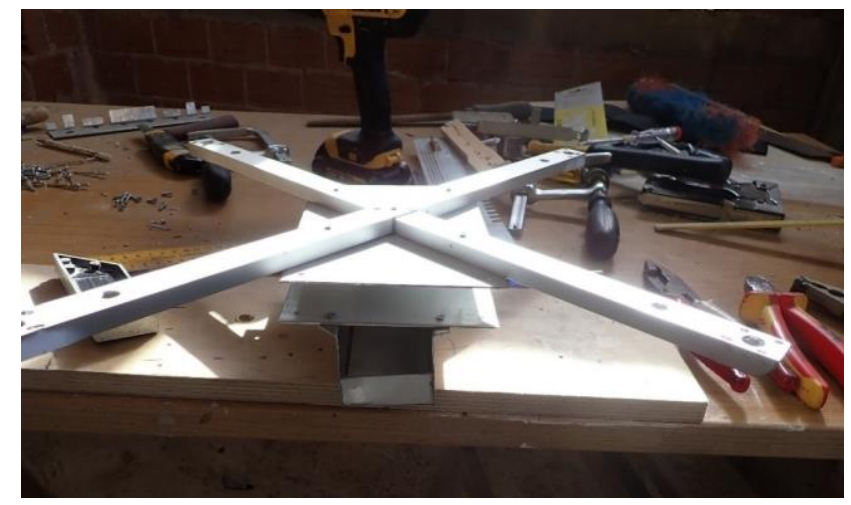

Fig. 1. Drone's Frame 


\section{B. Camera Mounting System}

For the Camera Mounting System, we used once again aluminium sheets. The camera is enclosed in a box for protection, placed on an axis so that it can move vertically. The axis is placed at the end of two arm like aluminium pieces, which in turn are screwed onto a round base. The base is pacted on a vertical axis so that it can rotate horizontally. The movement is achieved through two belts and the pulley located on every axis and onto the two servo motors.

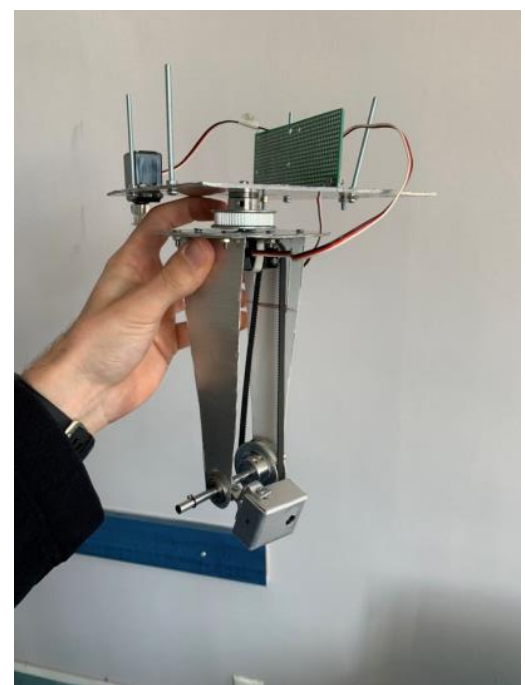

Fig. 2. Camera mounting System

\section{ELECTRONIC PARTS AND ALGORITHMS}

\section{A. Drone Parts}

Firstly, we used Arduino Uno as flight controller and the MultiWii as the flight algorithm. We altered the necessary code lines according to our sensors and set up and we also added a code routine for the collision avoidance part. As an accelerometer-gyroscope we used the MPU-6050, four 30amps electronic speed controllers, four motors $(700 \mathrm{kV})$ manufactured by SunnySky and the FlySky receiver (Papazoglou \& Lionis, 2015; Kalovrektis \& Katevas, 2019). As a power source, we used a lithium polymer battery $(14.8 \mathrm{~V}$, $50 \mathrm{c}, 6750 \mathrm{mAh})$ that was able to provide a flight autonomy of 20 minutes. Furthermore, we used two infrared sensors in order to measure the distance of an obstacle in front of and behind the drone, which are connected directly to the Arduino (Srinivasu \& Shanthi, 2015).

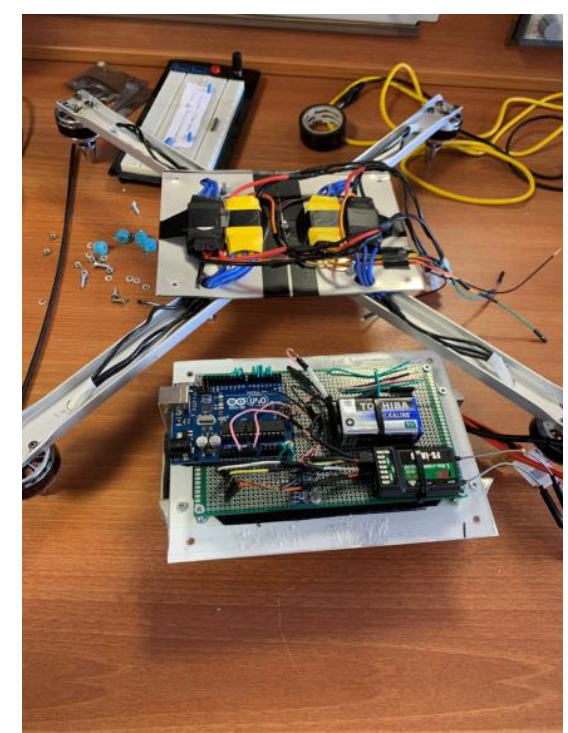

Fig. 3. Drone's electronic Circuit

\section{B. Mounting System Parts}

In order to achieve the horizontal and vertical movement of the camera we used servo motors as we previously mentioned. The motors are driven by the ESP-32 microcontroller which acts as a server and receives commands from a pc which acts as a client, via socket communication. There is a step-down converter so that we can power all the parts directly from the drone's main battery, and finally, we can receive the drone's coordinates through a GPS module that is also connected to the ESP-32 (Hernandez-Martinez et al., 2015). As for the camera, we chose the ESP-EYE which acts as a server too, and we can again connect to it as a client and have a live feed of the video.

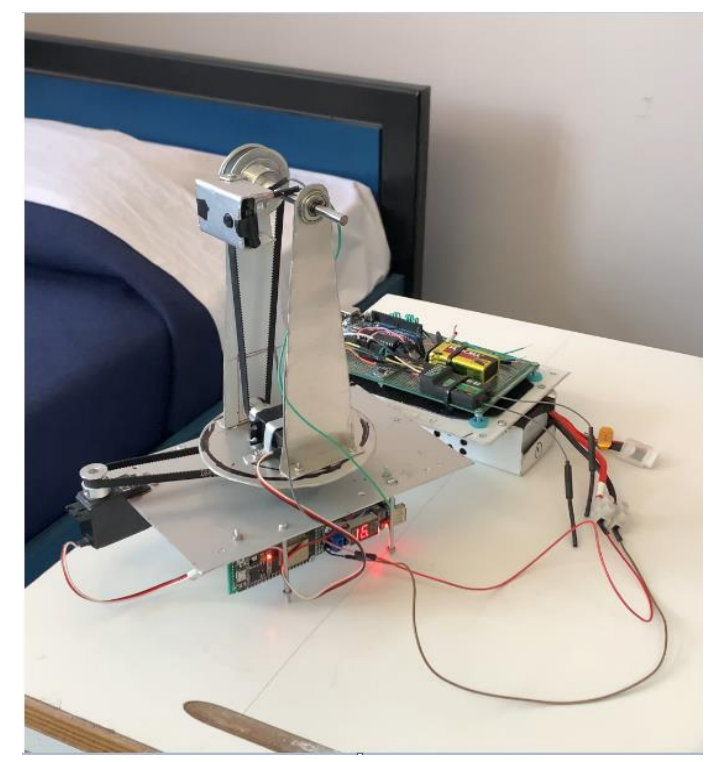

Fig. 4. Camera Powered by the drone's battery 


\section{Algorithms}

As mentioned above, we used an open source code as the flight algorithm (MultiWii v2.4) and we made the necessary modifications. The object tracking is mainly achieved trough the client-computer and the use of the open source libraries from the Open Computer Vision project (Open CV2). Therefore, we developed an algorithm using Python (Avouris, 2019; Chollet et al., 2018) which connects to the ESP-32 and at the same time streams the video feed from the ESP-EYE. The user has the total control of the camera in the beginning through the A, S, D, W and 'space' keys and as soon as he selects a target by using the trackbars and locking onto the desired color, by pressing 'ESC' the system will automatically track the object. More specifically, the algorithm converts the incoming frames from RGB to HSV color space, so that the user can easily choose the desired color and then it creates the contours corresponding to the user's choice. Afterwards in the tracking section, we set two centers, the first one is the center of the targeted object and the second is the center of the incoming image and the algorithm via the correlation of the two centers, sends the corresponding commands to the ESP-32 so that the two centers can meet (Rojas et al., 2017; (Hernandez-Martinez et al., 2015).

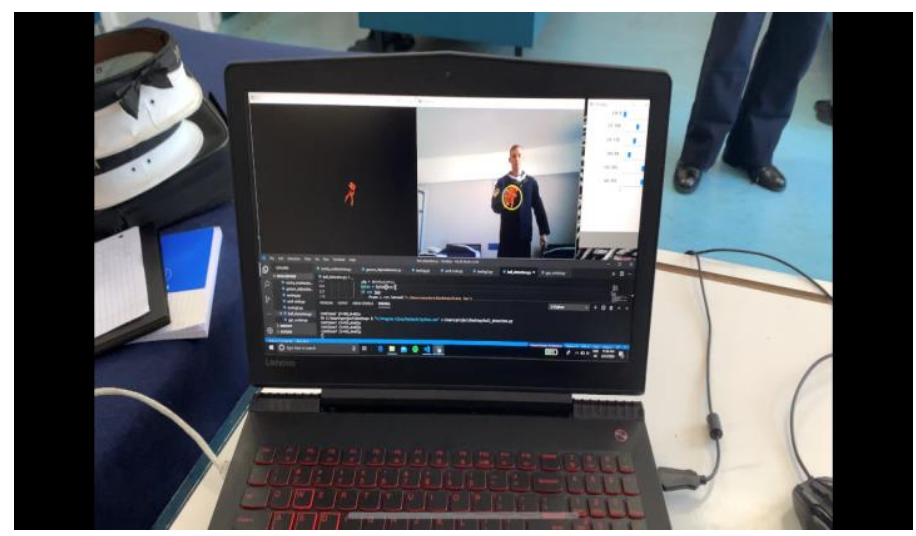

Fig. 5. Object Tracking Procedure

\section{TESTING AND RESULTS}

We performed several tests with and without the camera mounted onto the drone. The drone itself could hover for almost 20 minutes and the avoid collision system would not let it hit any obstacle even when the user tried to fly the drone towards it. Equipped with the camera system, the hover time decreased to just above 13 minutes as the weight of the construct increased significantly. The camera could track any desired object only if its color was distinctly different from the background due to the fact that the whole procedure is based on just the color of the targeted object and not on its shape, as well as the camera we used, had only 2Mpixels resolution. Finally, the pc user could control manually the camera and the
GPS module transmitted successfully the coordinates to him through the ESP-32, so every expectation was met to a certain degree.

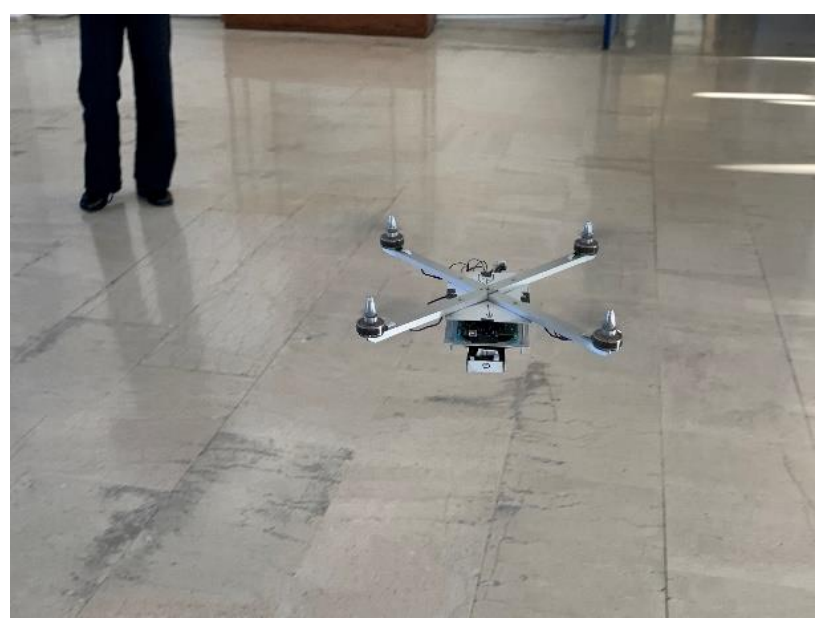

Fig. 6. Drone during take-off

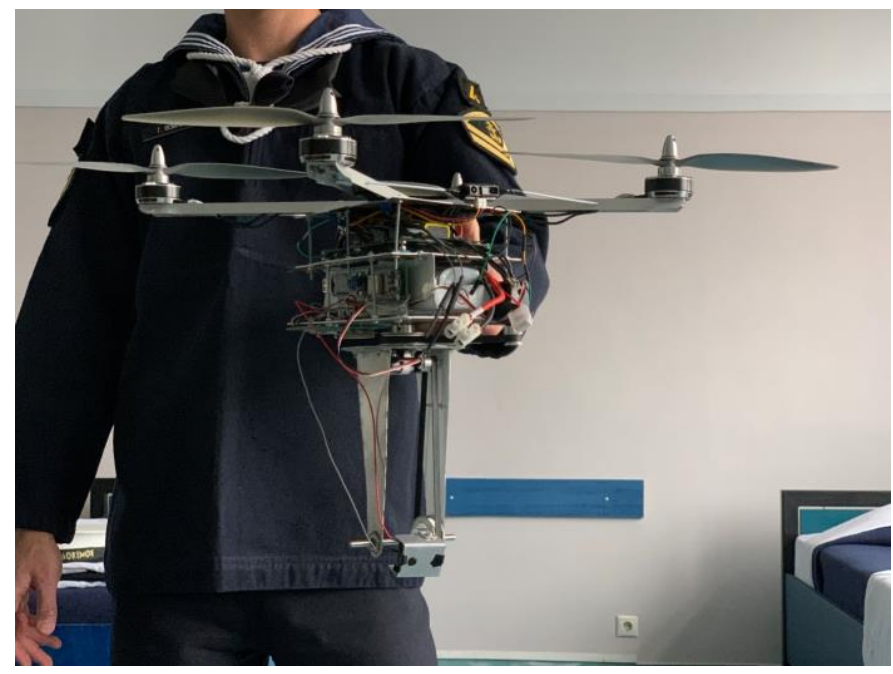

Fig. 7. The drone in its final form

\section{CONCLUSION}

We have presented the design and construction and demonstrated the successful operation of a UAV with GPS tracking, collision avoidance, surveillance via airorne camera and target tracking. There is always room for improvement of everything we do and this applies here as well. During the making and the testing it was realized that some things could be improved, for example instead of infrared sensors we could have used ulstrasonic ones. It is within our intentions to 
further improve the design and add sensors that will offer new functionalities.

\section{ACKOWLEDGMENT}

The authors acknowledge support from the Electronics Lab of the Hellenic Naval Academy.

\section{REFERENCE}

[1] Arduino IDE (Version 1.8.12) [Computer software]. (n.d.). Retrieved May 27, 2020, from https://www.arduino.cc/en/Main/Software

[2] Avouris, N. (2019). Introduction to Python. University of Patras, [online]. Retrieved from https://mathesis.cup.gr

[3] C++ Tutorial. (n.d.). Retrieved May 27, 2020, from https://www.w3schools.com/cpp/

[4] Chollet, F., Kalbermatter, S., Brownlee, J., Geitgey, A., Malisiewicz, T., Zdziarski, Z., . . . IP, P. (2018, September 19). You can master Computer Vision, Deep Learning, and OpenCV. Retrieved May 27, 2020, from https://www.pyimagesearch.com/

[5] ESP32 Overview | Espressif Systems. (n.d.). Retrieved May 27, 2020, from https://www.espressif.com/en/products/socs/esp32/over view

[6] Guilmartin, J. (2019, December 26). Unmanned aerial vehicle. Retrieved May 27, 2020, from https://www.britannica.com/technology/unmannedaerial-vehicle

[7] Hernandez-Martinez, E., Fernandez-Anaya, G., Ferreira, E., Flores-Godoy, J., \& Lopez-Gonzalez, A. (2015). Trajectory Tracking of a Quadcopter UAV with Optimal Translational Control. IFAC-PapersOnLine, 48(19), 226-231. doi:10.1016/j.ifacol.2015.12.038

[8] Jackson, B. A., Frelinger, D. R., Lostumbo, M. J., \& Button, R. W. (2008). Evaluating novel threats to the homeland: Unmanned aerial vehicles and cruise missiles. Santa Monica, CA: RAND National Defense Research Institute.

[9] Kalovrektis, K., \& Katevas, N. (2019). Aesthitires metrisis kai eleghou [Measurement and Control Sensors]. Thessaloniki, Greece: Tziolas Publications.

[10]Learn Python - Free Interactive Python Tutorial. (n.d.). Retrieved May 27, 2020, from https://www.learnpython.org/

[11]MultiWii (Version 2.4) [Computer software]. (n.d.). Retrieved May 27, 2020, from https://code.google.com/archive/p/multiwii/

[12]Papazoglou, P., \& Lionis, S. P. (2015). Anaptixi efarmogon me Arduino [Applications development with Arduino]. Thessaloniki, Greece: Tziolas Publications.
[13]Rojas, A. E., Espinal, A. A., Ramos, L., \& Aux, J. H. (2017). Design and construction of a prototype of an unmanned aerial vehicle equipped with artificial vision for the search of people. Sistemas Y Telemática, 15(41), 9-26. doi:10.18046/syt.v15i41.2453.

[14] Srinivasu, K., \& Shanthi, A. V. (2015). Designing a tote Quad copter, an Unmanned Aerial Vehicle (UAV). IJIREEICE, 3(4), 23-26. doi:10.17148/ijireeice.2015.3419. 